\section{Spontaneous Bacterial Peritonitis Caused by Bordetella hinzii}

\author{
Grace C. Wang, ${ }^{1}$ Miranda J. Wallace, ${ }^{1}$ \\ Gayathri Krishnan, Patrick D. Olson, \\ Abigail L. Carlson, ${ }^{2}$ Gautam Dantas, ${ }^{3}$ \\ James M. Fleckenstein ${ }^{3}$
}

Author affiliations: Saint Louis University School of Medicine, St. Louis, Missouri, USA (G.C. Wang); Washington University School of Medicine, St. Louis (M.J. Wallace, G. Krishnan, P.D. Olson,

A.L. Carlson, G. Dantas, J.M. Fleckenstein); St. Louis Veterans Affairs Health Care System, St. Louis (A.L. Carlson, J.M. Fleckenstein)

DOI: https://doi.org/10.3201/eid2711.211428

Although Bordetella hinzii coccobacilli is most commonly identified in respiratory tracts of birds and rodents, this organism has occasionally been isolated in human infections. We describe a case of $B$. hinzii spontaneous bacterial peritonitis in Missouri, USA. Whole-genome sequencing of blood and peritoneal fluid isolates confirmed B. hinzii infection.

$B$ ordetella hinzii is a gram-negative aerobic coccobacilli respiratory pathogen in poultry (1) and rodents (2). Human infections are rare but occur in immunocompromised persons (3) or upon exposure to infected animals. Most reported human infections are pulmonary; however, other manifestations include cholangitis (4) and periaortic abscess (5). We report a case of $B$. hinzii spontaneous bacterial peritonitis (SBP) complicated by bacteremia.

A 71-year-old man with alcoholism, hepatitis C, and decompensated cirrhosis, on day 28 of a 28 -day regimen of intravenous vancomycin for Streptococcus salivarius bacteremia and SBP, underwent outpatient paracentesis. After paracentesis (7.8 L of fluid removed), the patient experienced hypotension and orthostasis, which resolved after intravenous albumin, and returned home. He later sought care at an emergency department for weakness, abdominal pain, hypotension $(68 / 42 \mathrm{~mm}$ $\mathrm{Hg}$ ), and tachycardia (heart rate 130 beats/min).

\footnotetext{
${ }^{1}$ These first authors contributed equally to this article.

${ }^{2}$ Current affiliation: Centers for Disease Control and Prevention, Atlanta, Georgia, USA.

${ }^{3}$ These authors contributed equally to this article as co-principal investigators.
}

Despite intravenous fluid and albumin $(1.5 \mathrm{~g} /$ $\mathrm{kg}$ ) resuscitation, daptomycin, and piperacillin/azobactam, the patient experienced septic shock and hepatorenal syndrome, necessitating pressors. We substituted meropenem for piperacillin/tazobactam. On day 3, we discontinued daptomycin; administered albumin $(1 \mathrm{~g} / \mathrm{kg})$; and initiated octreotide, midodrine, and rifaximin. We discontinued pressors on day 4 . The patient improved clinically and his SBP resolved, as indicated by results of serial peritoneal fluid studies. On day 12, we replaced meropenem with ertapenem. After discharge (day 15), the patient completed 2 weeks of ertapenem, followed by daily trimethoprim/sulfamethoxazole prophylaxis.

Further investigation revealed the patient had a dog with cough and a cat with gastroenteritis, and neither pet was receiving veterinary care. His wife maintained several birdfeeders, but she had no symptoms. Five months after seeking care, the patient died after cardiac arrest and transjugular intrahepatic portosystemic shunt occlusion.

Initial ascites fluid studies revealed 1,673 leukocytes with $80 \%$ segmented neutrophils. Peritoneal fluid and blood cultures from day 1 of hospitalization had tiny oxidase-positive, indole-negative, gramnegative rods that grew on blood and chocolate agars but not MacConkey or CNA agars. After tentatively identifying the organism as a Bordetella species, we performed limited antimicrobial susceptibility testing for levofloxacin $(1.5 \mu \mathrm{g} / \mathrm{mL})$, ertapenem $(0.19$ $\mu \mathrm{g} / \mathrm{mL}$ ), meropenem (sensitive), and trimethoprim/ sulfamethoxazole $(0.004 \mu \mathrm{g} / \mathrm{mL})$. The Missouri State Public Health Laboratory (Jefferson City, MO, USA) later confirmed identification as $B$. hinzii by using matrix-assisted laser desorption/ionization time-offlight mass spectrometry.

Blood and peritoneal fluid isolates underwent whole-genome sequencing (WGS) to confirm identification. We suspended isolate plate scrapes in Luria-Bertani broth with 15\% glycerol and stored them at $-80^{\circ} \mathrm{C}$. After thawing $250-500 \mu \mathrm{L}$ of each suspension, we extracted genomic DNA by using the QIAamp BiOstic Bacteremia DNA Kit (QIAGEN, https://www.qiagen.com). We used $0.5 \mathrm{ng}$ of genomic DNA to prepare sequencing libraries with the Nextera XT DNA Library Preparation Kit (Illumina, https://www.illumina.com). We pooled and sequenced libraries on the NovaSeq 6000 platform (Illumina) to obtain $\approx 5$ million $2 \times 150$ bp reads. We used Trimmomatic 38.0 (https://github.com/ timflutre/trimmomatic) to demultiplex the reads 
and remove adaptors. We removed contaminating reads by using Deconseq4.3 (http:/ / deconseq.sourceforge.net) and repaired disordered reads by using BBTools Repair (BBTools 38.26; https://jgi.doe.gov/ data-and-tools/bbtools). We assembled de novo genomes by using the Unicycler 0.4.7 Illumina-only assembly process and then annotated by using Prokka 1.14.5 (https://github.com/tseemann/prokka). We determined assembly quality by using QUAST 4.5 (http://quast.sourceforge.net) and checkM 1.0.13 (https://github.com/Ecogenomics/CheckM).

We performed pairwise average nucleotide identity between the isolates and deposited Bor- detella genomes by using pyani (https://github. $\mathrm{com} /$ widdowquinn/pyani). We performed coregenome alignments by using roary 3.12 .0 (https:/ / sanger-pathogens.github.io/Roary) and then generated approximate maximum-likelihood trees on the basis of the roary alignment file by using FastTree 2.1.7 (http://www.microbesonline.org/fasttree). We identified single-nucleotide polymorphisms (SNPs) by using Snippy 4.4 .3 (https://anaconda. org/bioconda/snippy/files) and antimicrobialresistance genes by using AMRFinder 3.8.4 (https:/ / github.com/ncbi/amr). We deposited raw sequence data and genomic assemblies to the National

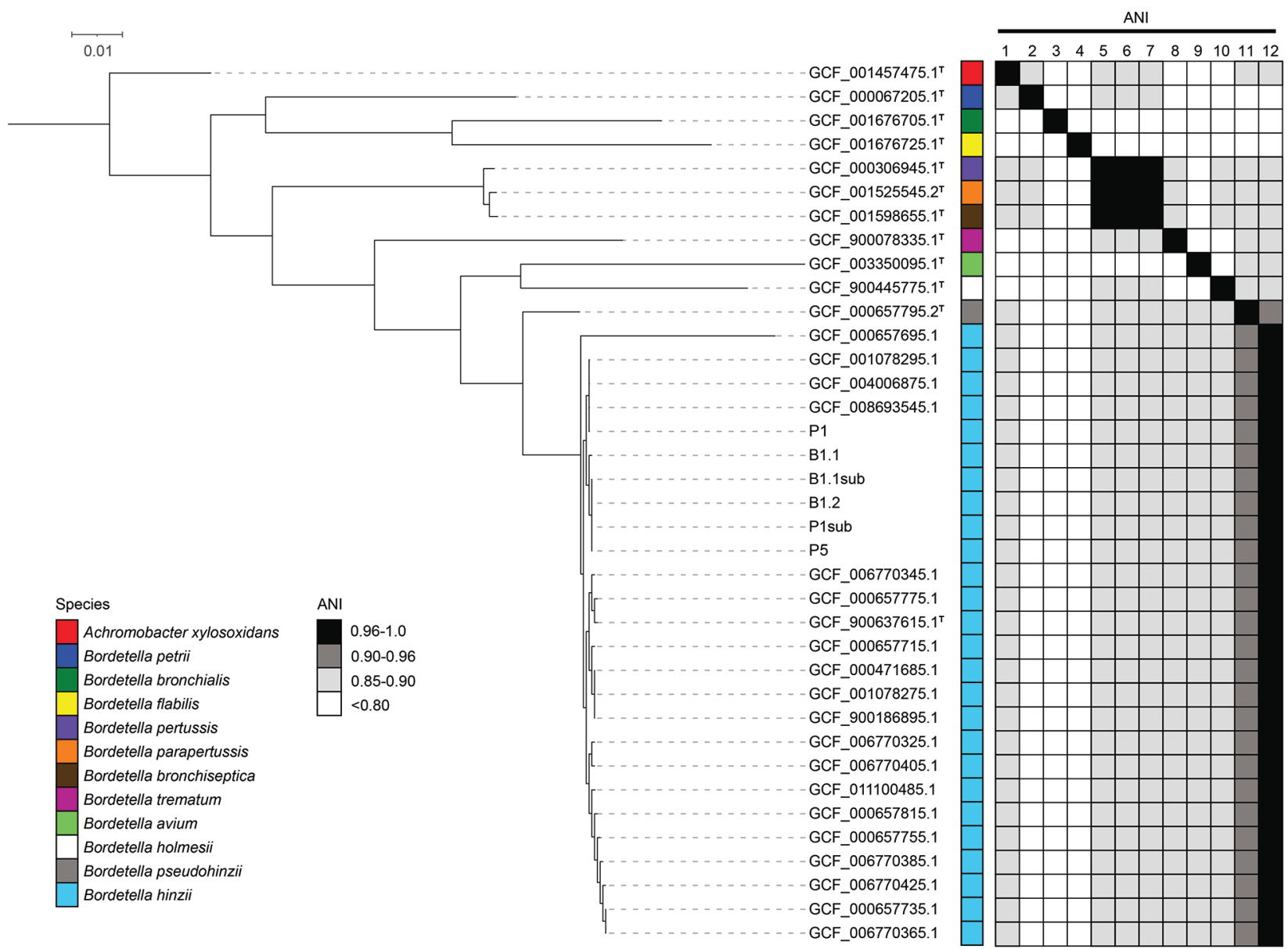

Figure. Comparative genomic analyses of Bordetella hinzii isolates from a patient in Missouri, USA, with type and nontype Bordetella assemblies. After core-genome alignment (58 total core genes), a neighbor-joining phylogenetic tree rooted with Achromobacter xylosoxidans as the outgroup demonstrates the isolates from this study cluster with other previously deposited $B$. hinzii genomes. Pairwise ANI was performed against type assemblies. The isolates in this study meet the ANI threshold ( $\geq 0.96 \%)$ for species-level identity with $B$. hinzii type assembly GCF_900637615.1 (7). Isolates were recovered from peritoneal fluid cultures collected at day 1 and day 5 (P1 and P5, respectively; P2sub is a subculture of P1). Blood isolates were recovered from blood cultures collected on day 1 (B1.1 and B1.2; B1.1sub is a subculture of B1.1). As previously observed (8), the type genomes for B. pertussis, B. parapertussis, and B. bronchiseptica represent an instance of previously established, distinct species that exceed the species-level ANI threshold relative to each other. ${ }^{\top}$ indicates assemblies generated from type material. Type assemblies are numbered 1-12 on vertical axes as follows: 1 , GCF_001457475.1; 2, GCF_000067205.1; 3, GCF_001676705.1; 4, GCF_001676725.1; 5, GCF_000306945.1; 6, GCF_001525545.2; 7, GCF_001598655.1; 8, GCF_900078335.1; 9, GCF_003350095.1; 10, GCF_900445775.1; 11, GCF_000657795.2; 12, GCF_900637615.1. ANI, average nucleotide identity. 
Center for Biotechnology Information (BioProject no. PRJNA706405).

We performed Illumina short-read WGS on $6 \mathrm{pu}-$ tative $B$. hinzii isolates recovered from peritoneal fluid and blood cultures from day 1 and a peritoneal fluid culture from paracentesis on day 5. Altogether, the isolate assemblies had an average length of $4.8 \mathrm{Mbp}$ (range 4.70-4.84 Mbp) and GC content of $\approx 67.2 \%$, reflective of published $B$. hinzii genomes (6). We built a neighbor-joining phylogenetic tree by using a core-genome alignment of the isolates with publicly available Bordetella genomes. The isolates formed a clade with $B$. hinzii genomes, including a type that was distinct from other Bordetella species. Pairwise average nucleotide identity analysis showed the isolates meet the specieslevel threshold ( $\geq 96 \%)$ (7) exclusively with genomes originating from $B$. hinzii (Figure). SNP analyses within the 6 isolates suggested they were clonal because $\leq 2$ SNPs (all nonsynonymous) were found between each strain pair, further confirming the clinical laboratory indications that the isolates are B. hinzii and that organisms recovered from peritoneal sites and blood originated from the same source. In addition, we identified a putative novel $\beta$-lactamase gene with $51 \%$ identity to the class-A LRA-1 $\beta$-lactamase (Comprehensive Antibiotic Resistance Database [https://card.mcmaster. ca/home]; accession no. ARO:3002482). This gene is likely endogenous because it appeared in all available B. hinzii assemblies.

In summary, WGS of blood and peritoneal fluid isolates confirmed a set of clonal B. hinzii isolates from both tissue types from this patient. Our findings provide compelling evidence for serious human infection caused by this organism.

\section{Acknowledgments}

We thank Paul Weiland, Mary Ann Smith, and Joan Lorenz; Kimberley V. Sukhum for thoughtful review of this manuscript; and the staff of the Genome Technology Access Center at the McDonnell Genome Institute for their sequencing services.

This work was supported in part by awards to G.D. through the National Institute of Allergy and Infectious Diseases of the National Institutes of Health (grant no. U01AI123394), the Agency for Healthcare Research and Quality (grant no. R01HS027621), and the Congressionally
Directed Medical Research Program of the US Department of Defense (grant no. W81XWH1810225). This work also was supported by a T32 fellowship award granted to M.J.W. through the National Cancer Institute of the National Institutes of Health (award no. T32 CA113275-12).

\section{About the Author}

Dr. Wang is a resident physician in the Department of Internal Medicine at Saint Louis University School of Medicine. She is interested in both the clinical and translational research aspects of pulmonology and critical care medicine, as well as drug discovery and mechanisms of disease.

\section{References}

1. Register KB, Kunkle RA. Strain-specific virulence of Bordetella hinzii in poultry. Avian Dis. 2009;53:50-4. https:/ / doi.org/10.1637/8388-070108-Reg.1

2. Jiyipong T, Morand S, Jittapalapong S, Raoult D, Rolain JM. Bordetella hinzii in rodents, Southeast Asia. Emerg Infect Dis. 2013;19:502-3. https://doi.org/10.3201/eid1903.120987

3. Cookson BT, Vandamme P, Carlson LC, Larson AM, Sheffield JV, Kersters K, et al. Bacteremia caused by a novel Bordetella species, "B. hinzii". J Clin Microbiol. 1994;32:2569_ 71. https://doi.org/10.1128/jcm.32.10.2569-2571.1994

4. Arvand M, Feldhues R, Mieth M, Kraus T, Vandamme P. Chronic cholangitis caused by Bordetella hinzii in a liver transplant recipient. J Clin Microbiol. 2004;42:2335-7. https://doi.org/10.1128/JCM.42.5.2335-2337.2004

5. González MM, Romano MPC, de Guzmán García Monge MT, Martín BB, García AS. Bordetella hinzii endocarditis, a clinical case not previously described. Eur J Case Rep Intern Med. 2019;6:000994.

6. Weigand MR, Changayil S, Kulasekarapandian Y, Tondella ML. Complete genome sequences of two Bordetella hinzii strains isolated from humans. Genome Announc. 2015;3:1-2. https:/ / doi.org/10.1128/genomeA.00965-15

7. Richter M, Rosselló-Móra R. Shifting the genomic gold standard for the prokaryotic species definition. Proc Natl Acad Sci U S A. 2009;106:19126-31. https:/ / doi.org/10.1073/ pnas.0906412106

8. Kim M, Oh HS, Park SC, Chun J. Towards a taxonomic coherence between average nucleotide identity and $16 \mathrm{~S}$ rRNA gene sequence similarity for species demarcation of prokaryotes. Int J Syst Evol Microbiol. 2014;64:346-51. https:/ / doi.org/10.1099/ijs.0.059774-0

Address for correspondence: James M. Fleckenstein, Division of Infectious Diseases, Washington University School of Medicine, 660 S Euclid Ave, Campus Box 8051, St. Louis, MO 63110, USA; email: jfleckenstein@wustl.edu 